\title{
Medienbildung als Prozess der Unsgestaltung zwischen formaler Medienerziehung und informeller Medienaneig- nung
}

\begin{abstract}
Ausgehend von aktuellen medialen Entwicklungen und Veränderungen der Mediennutzungsmuster von Jugendlichen versucht der Artikel den Medienbildungsbegriff systematisch mit den Komplexen Medienerziehung, Medienaneignung, Medienliteralität und Medienkompetenz zu verbinden, indem Medienbildung mit jeweils zwei dieser Komplexe in Beziehung gesetzt wird. Als zentrales Ergebnis dieser Betrachtung scheint es notwendig, den Individuen-zentrierten und vorwiegend institutionalisierten Ansatz der Selbstgestaltung innerhalb der Medienbildung um vielfältige und informelle Formen selbstorganisierten sozialen Lernens in Online-Vergemeinschaftungen wie Social Networks zu erweitern. Beim Medienhandeln in einer partizipativen und kollektiven (Online-)Kultur geht es nicht mehr vornehmlich um die Gestaltung des eigenen Selbst, sondern um eine relationale und gemeinsame Gestaltung im Sinne einer Unsgestaltung der Teilnehmenden.
\end{abstract}

\section{Einleitung}

Der Begriff der Medienbildung hat sich im medienpädagogischen Diskurs zunehmend etabliert. Damit entsteht ein neuer Schlüsselbegriff, der einer Verortung, Einordnung und Abgrenzung zu bisherigen Konzepten wie Medienerziehung und Medienaneignung, aber auch zu Zielgrößen wie Medienkompetenz und Medienliteralität, bedarf. Vor dem Hintergrund aktueller Mediennutzungsmuster und eines damit einhergehenden praxeological turns (Hirschauer, 2004, S. 89) ist u.E. eine Erweiterung bisheriger Definitionsversuche um eine relational-kollektive Perspektive notwendig.

Ein zentrales Modell zur Medienbildung stammt von Winfried Marotzki und Benjamin Jörissen, die im Sinne der strukturalen Bildungstheorie Medienbildung als selbstreflexiven Lern- und Orientierungsprozess verstehen, in welchem "vorhandene Strukturen und Muster der Weltaufordnung durch komplexe Sichtwei- 
sen auf Welt und Selbst ersetzt werden" (Jörissen \& Marotzki, 2008, S. 100). Der Kern des Medienbildungsgedankens nach Marotzki und Jörissen ist die "Notwendigkeit, die wachsende Unbestimmtheit und zunehmende Komplexität der Moderne mit den Mitteln gesteigerter medialer Reflexivität zu bearbeiten." (Jörissen \& Marotzki, 2009, S. 240).

Auch für Heinz Moser bezeichnet Medienbildung die Verarbeitung und Integration von Informationen aus subjektunabhängigen Datennetzen und Informationssystemen in den eigenen Lebens- und Erfahrungskontext (Moser, 2010, S. 314f). Er integriert dabei die konstruktivistische Perspektive einer Selbsterzeugung von Ich und Welt im Kontext einer reflexiven Verständigung.

Für Dieter Spanhel ist Medienbildung ein selbstgesteuerter Prozess der "wechselseitige[n] Erschließung von Mensch und Welt in der aktiven Auseinandersetzung mit der Vielfalt der Medien und der Fülle an Medienangeboten", ist notwendigerweise an Kommunikationsprozesse gebunden und kein "kausalmechanistischer Zusammenhang" (Spanhel, 2006, S. 188ff).

Ben Bachmair versteht Medienbildung als einen Prozess, "innerhalb dessen Subjekte vorgegebenen Inhalten eigene Bedeutungen verleihen" (Bachmair, 2009, S. 177). Zentral ist für ihn, dass sich Bildung in der reflexiven Spannung zwischen der Aneignung kultureller Objekte und der Artikulation bzw. Entäußerung im Sinne einer Umgestaltung von Welt und eines Sich-in-Beziehung-Setzens zur Welt entfaltet:

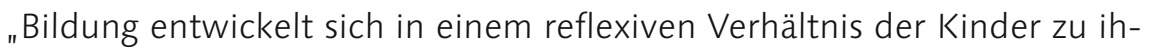
rer sozialen, kulturellen und dinglichen Umwelt und zu ihrer emotionalen und kognitiven Innenwelt. Die Aneignung kultureller Manifestationen bietet Kindern die Chance sich zu entfalten, sich als Subjekt in Beziehung zur kulturellen Umwelt zu setzen, in der Umwelt ihre Spuren zu hinterlassen und dabei die Umwelt zur eigenen Lebenswelt zu verbinden und umzugestalten. Die reflexive Spannung zu den kulturellen Produkten und der sie tragenden Umwelt bietet Chancen der Kritik und Distanz, die in eine reflektierte und abwägende Form der Teilhabe und Gestaltung münden können." (ebenda, S. 227)

Konstitutiv für diese Definitionen ist, dass der Bildungsbegriff als Selbstgestaltungsprozess zu verstehen ist. Darauf aufbauend soll in diesem Beitrag zunächst die Frage gestellt werden, warum Bildung in eine spezielle Medienbildung untergliedert werden soll (Kapitel 2). Daran anschließend wird der Begriff der Medienbildung zunächst auf der Ebene der Methode in Bezug zur Medienerziehung (im Sinne einer institutionalisierten Unterstützung, Förderung und Ver- 
mittlung) und Medienaneignung (Entwicklung, Entfaltung, Sozialisation vor dem eigenen sozio-kulturellen Lebenshintergrund) gesetzt (Kapitel 3; Abbildung 1, Feld 1). Anschließend wird in Kapitel 4 auf der Ebene der Ziele und Inhalte die Medienbildung von Medienkompetenz und Medienliteralität (media literacy) abgegrenzt werden (Abbildung 1, Feld 2). In Kapitel 5 ist zu klären, welche Orientierungsfunktion und Möglichkeiten der personalen Selbstgestaltung Medienbildung sowohl in der klassisch formalen, schulisch-curricularen Struktur (Begriffspaar Medienerziehung und Medienkompetenz; Abbildung 1, Feld 3) als auch in informellen und deinstitutionalisierten Prozessen des selbstorganisierten sozialen Lernens in Peer Gruppen, Vergemeinschaftungen wie z.B. Diasporas oder Szenen (Begriffspaar Medienaneignung und Medienliteralität; Abbildung 1, Feld 4) erfährt. Dabei wird herausgearbeitet, dass sich der Medienbildungsbegriff nicht mehr nur auf die Selbstgestaltung des Subjektes, sondern auch auf die Unsgestaltung der Teilnehmenden partizipativer und kollektiver (online-)Kulturen bezieht.

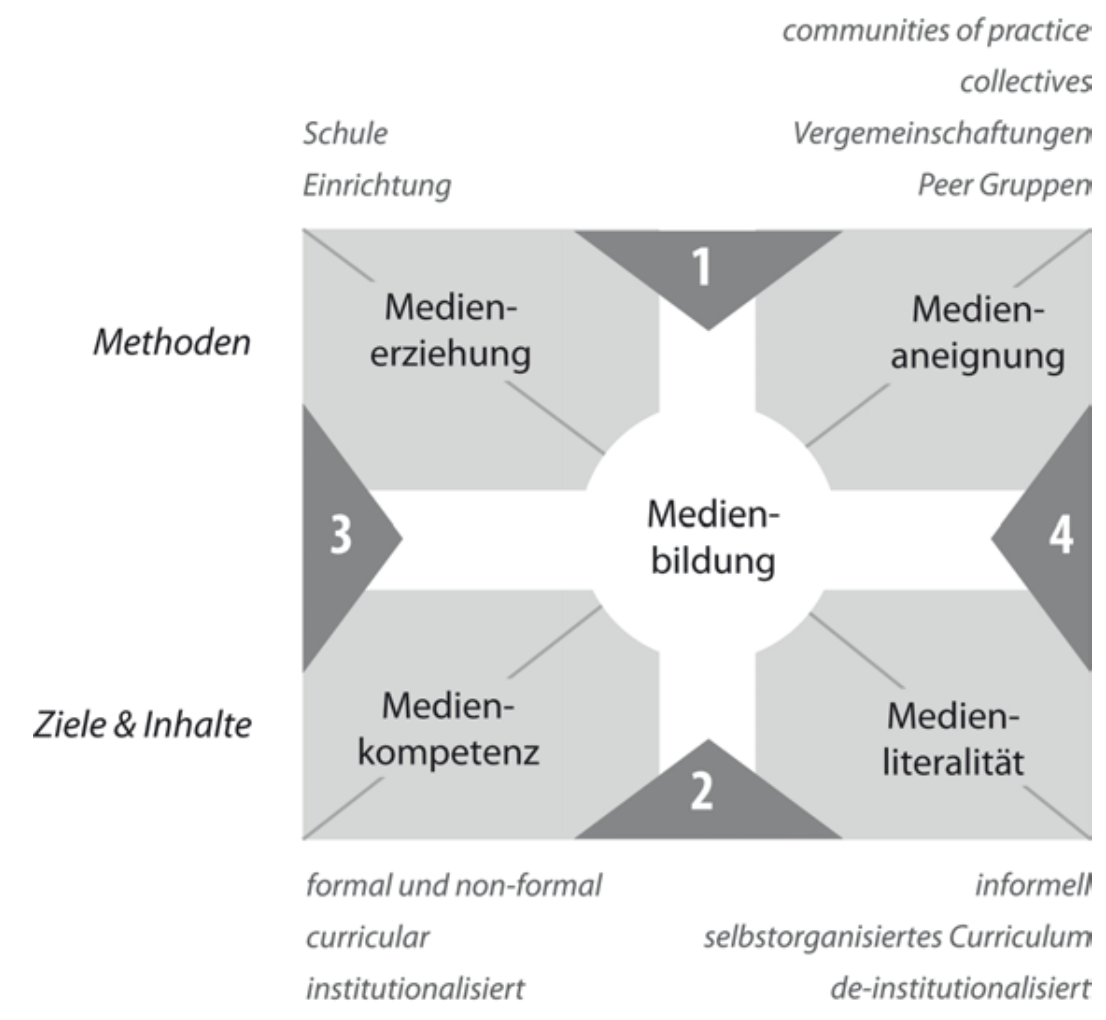

Abb. 1: Handlungsfelder der Medienbildung 


\section{Notwendigkeit der Medienbildung durch Mediatisierung?}

Grundsätzlich stellt sich die Frage, wieso es eines spezifischen "Medien"bildungsbegriffes überhaupt bedarf. Erfährt Bildung - verstanden als kreative Selbstgestaltung des Menschen als Person, also als "frei verantwortliches Subjekt in einem geschichtlichen Prozess unter den einschränkenden Bedingungen von Raum und Zeit" (Böhm, 1995, S. 152) - durch die mediale Unterstützung eine spezifische Umformung oder zumindest eine zusätzliche Akzentuierung?

Ein Argument dafür könnte die Etablierung des globalen digitalen Datennetzes Internet und der damit einhergehenden grundlegenden Veränderungen von Informations- und Kommunikationsprozessen sein. Dieses unter dem Begriff der Mediatisierung gefasste Phänomen (siehe Krotz, 2007) zieht weitreichende Transformationen, auch bei den bildungsbezogenen Handlungen und Interaktionen der jeweiligen Akteure, sowie der durch sie emergierenden Systeme (Wolf, 2010) - in der Dialektik von Habermas über ein rein technisches und praktisches Interesse hinausgehend mit emanzipatorischem Potenzial - auf vier unterschiedlichen Dimensionen nach sich:

(a) Soziale Dimension: Das Internet ermöglicht eine hohe Individualisierung der Lernprozesse und -inhalte, da persönliche Lerninteressen in idiosynkratischen Curricula beliebig realisiert werden können. Dies löst zunehmend staatliche Normvorgaben ab. Gleichzeitig entsteht durch die Vernetzung (z.B. in einer fachlichen Blogosphäre) eine neue Form der Vergemeinschaftung, die gegebenenfalls eine neue Normierung und Standardisierung von Inhalten und Kompetenzen etabliert, welche selbstorganisiert einer beliebigen Individualisierung entgegenwirkt.

Aus einer personalistischen Perspektive entsteht ein Raum für Freiheit und Selbständigkeit der menschlichen Person. Diese frei zu realisierende Berufung innerhalb einer (personalen) Gemeinschaft bedarf eines eigenverantwortlichen Engagements, welches ohne entsprechende Medienbildung kaum realisierbar erscheint.

(b) Räumliche Dimension: Das globale Datennetz führt zur Deterritorialisierung, also Abkopplungsprozessen kultureller Formen von einzelnen Territorien. In den Mittelpunkt dieser Betrachtung rücken spezifische Kommunikationsräume und Formen von Wissensspeicherung und -verteilung, aber auch von kritischer Reflexion und emanzipatorischer Koalition. Gemeinsames Arbeiten und Lernen wird plötzlich auch bei räumlicher Distanz ermöglicht. Das Erreichen einer kritischen Masse zu beliebig speziellen Fragestellungen und Interessen wird erleichtert, eigene nichtterritoriale Lern- und Bildungskulturen können sich entwickeln, der Zugang wird erleichtert. 
(c) Zeitliche Dimension: Die modernen Kommunikationskanäle forcieren eine zeitliche Verdichtung von Diskursen und Erfahrungen. So werden z.B. Antwortzyklen durch E-Mails, Instant Messaging, Chats, Blogkommentare oder Microblogging zunehmend verkürzt. Dies kann Meinungsbildung und Weiterentwicklung fördern, birgt aber auch die Gefahr einer mangelnden Tiefe von Reflexion.

(d) Eine zusätzliche Perspektive der Mediatisierung ist in Ergänzung zu Krotz die ökonomische Dimension: Die Entstofflichung der Medien (z.B. vom Buch zum PDF oder von der CD zum MP3) führt zu nahezu kostenfreien Distributions- und Lagerprozessen. Dies zieht mittelfristig - zumindest in Themenbereichen des allgemeinen Interesses - durch Nutzung des cognitive surplus (Shirky, 2010) und im Zuge der Open Content Bewegung ein Überangebot kostenloser Angebote nach sich. Folglich wird von einer Aufmerksamkeitsökonomie gesprochen (Goldhaber, 1997) - das knappe Gut sind die Lernenden, die sich mit einem Thema lernend beschäftigen wollen, nicht die frei verfügbaren Lehrmaterialien. Wurden früher öffentliche Bibliotheken in der Absicht errichtet, den unterprivilegierten Schichten einen Grundzugang zu Wissensressourcen zu eröffnen, wird dieser Zugang über Open Access, Google Books und illegale Downloads nun nahezu allumfassend. Diesem Überangebot von Wissens- und Lehrkonserven steht aber ein weiterhin limitiertes Angebot qualitativ hochwertiger Unterstützungsprozesse des Lernens durch Lehrende gegenüber - und gegebenenfalls auch einem Mangel an Bildungsnähe.

So wie in der Musikindustrie plötzlich das Konzert als performance wieder den eigentlichen Wert bildet, da in seiner sinnhaften Erfahrung nicht herunterladbar, so bleibt in Lehr-Lern-Prozessen die persönliche Unterstützung durch andere Mitlernende oder Lehrende nicht nur zentrale Gelingensbedingung und Qualitätskriterium, sondern ist Grundlage jedweder personaler Bildungsprozesse. Je weniger Orientierungswissen und Bildsamkeit entwickelt ist, desto weniger können erweiterte Zugänge genutzt werden.

\section{Erstes Zwischenfazit: Mediatisierung von Bildungsprozes- sen als cultural turn}

Zentral für Bildungsprozesse erscheint die soziale Dimension der Mediatisierung, die durch räumliche und zeitliche Aspekte weiter verdichtet wird und neue Zugänge zu Bildungsressourcen schafft. Das Internet verstärkt die Selbstgestaltungsmöglichkeiten der Person, da Kommunikation bzw. darauf aufbauend gemeinsame Gestaltung (collaboration) in den Mittelpunkt dieses Mediums rückt. Dies ändert die Fokussierung von medial unterstützten Bildungsprozessen: waren technische Medien des Bildungsmainstreams vor allem Lehrmedien (Buch, Tafel, 
Overheadprojektor, Beamer), so öffnet das Internet in seiner Mitmachform neue kommunikative und gestalterische Lern-, Bildungs- und Entwicklungsräume.

\section{Medienbildung zwischen Medienerziehung und Medien- aneignung}

Die Medienpraxis der heranwachsenden Generation entwickelt sich zwischen einer normativ orientierten und institutionalisierten Medienerziehung und einer selbstorganisierten Medienaneignung (Abb. 1, Feld 1).

Medienaneignung findet im sozio-kulturellen Alltag der Jugendlichen, bspw. in der Gemeinschaft mit der Peer Gruppe, statt. Etwa drei Viertel der Jugendlichen von 12-19 Jahren nutzen Online Communities (Feierabend \& Rathgeb, 2009, S. 45), von den Social-Web-Angeboten steht YouTube für alle Jugendlichen an erster Stelle (Schmidt, Rohde, \& Hasebrink, 2009, S. 22) und etwa die Hälfte der Jugendlichen machen Fotos und Filme mit ihrem Handy (ebenda, S. 12).

Aber dennoch scheint sich eine Medienbildung en passant gerade nicht zu entwickeln, wie Rolf Schulmeister am Beispiel der These der digital natives bzw. 'Net Generation' detailliert erörtert (vgl. Schulmeister, 2009). So besteht weiterhin ein Bedarf für die Medienerziehung: sowohl vor dem Hintergrund negativer Phänomene der neuen Netzkultur wie z.B. Cyber-Bullying aber auch der Schlüsselfunktion des Internets für die Partizipation in allen Lebensbereichen.

\subsection{Medienerziehung}

Die Medienerziehung ist der formale und institutionalisierte Teil der Medienbildung innerhalb der Profession Medienpädagogik. Die Medienerziehung umfasst Bemühungen und Reflexionen in institutionellen Lernumfeldern, „....die das Ziel haben, ein humanes bzw. verantwortliches Handeln im Zusammenhang mit der Mediennutzung und Mediengestaltung zu entwickeln" (Tulodziecki, 2008, S. 110). Nach Gerhard Tulodziecki geht es bei Medienerziehung um die Anregung und Unterstützung reflektierter „....Nutzung vorhandener Medienangebote und die eigene Gestaltung von Medienbeiträgen im Sinne kommunikativer Kompetenz und sozialen Handelns" (ebenda, S. 111). Für jedes dieser beiden Felder der Nutzung und Produktion gilt es im Rahmen der Medienerziehung folgende drei Inhalts- und Reflexionsfelder umzusetzen:

- "Verstehen und Bewerten von Mediengestaltungen"

- "Erkennen und Aufarbeiten von Medieneinflüssen"

- "Durchschauen und Bewerten von Bedingungen" (ebenda, S. 112). 
Tulodziecki geht demnach zunächst von der Nutzung bestehender Angebote aus, die dann in die eigene Gestaltung von Medienangeboten münden soll.

Einen ähnlichen Ansatz verfolgt der britische Medienpädagoge David Buckingham, der einen engen Vermittlungszusammenhang zwischen Erziehung und der kulturellen Handlungsfähigkeit von Kindern und Jugendlichen sieht (vgl. Bachmair \& Burn, 2009, S. 135). Diese Handlungsfähigkeit gilt es im situativen Rahmen der Schule zu fördern, „....was bei der kulturellen Praxis von Kindern bzw. Jugendlichen ansetzt und deren kreative Mediennutzung kritisch weiterentwickelt" (ebenda, S. 135). Indem Buckingham die kulturelle Praxis der Medienaneignung und die sich darin entwickelnde Alltagsmedienkompetenz der Kinder und Jugendlichen anerkennt, sieht er eine der neuen Aufgaben der Medienpädagogik darin, diese informellen Lernprozesse aus dem Alltag für Medienerziehung in der Schule zu erschließen (vgl. Bachmair \& Burn, 2009, S. 135; Seipold, Rummler, \& Rasche, 2010, S. 227).

Medienerziehung in pädagogisch-erzieherischen Kontexten wie der Schule bildet in diesem Sinne den methodischen Zugang für die Förderung des Prozesses der Entwicklung von Medienbildung im Kontext institutionaler und formaler Bildungsprozesse.

Buckinghams Ansatz der Medienerziehung setzt dafür zentral auf die "Kreativität als Maßstab einer praktischen Medienarbeit, mit deren Hilfe sich kritischtheoretische Zugänge zu Medien erfahrbar machen lassen... [...] Kreativität hat somit eine Vermittlungsfunktion zwischen der unkritischen kulturellen Erfahrung und der Anwendung kritischer Konzepte" (Bachmair \& Burn, 2009, S. 133).

\subsection{Medienaneignung}

Aus der Medienforschung der Cultural Studies und dem Konzept des Kreislaufs der Kultur (Johnson, 1999) ist der Ansatz hervorgegangen, Medienkonsum als einen Gesamtprozess der kulturell kontextualisierten Aneignung aufzufassen (Hepp, 2005, S. 67). Unter Aneignung (engl. "appropriation“) versteht Hepp das „...Sich- zu-Eigen-Machen' von (Medien-) Produkten“ (ebenda, S. 68). Der Kernpunkt innerhalb des Prozesses der Aneignung ist der reflexive Bezug auf die eigene Alltagswelt und Lebenswirklichkeit. Diesem Verständnis von Aneignung nach finden Rezeption, Nutzung und Interpretation von Medien auch in einer zunehmend globalisierten Medienkommunikation nicht losgelöst von der menschlichen Lebenswelt statt (vgl. ebenda, S. 71). Demzufolge lässt sich der Mediennutzungsprozess nicht allein mit eindimensionalen Konzepten der Medienwirkung oder Manipulation beispielsweise im Sinne der Lasswell-Frage "Wer sagt was zu wem mit welcher Wirkung", beschreiben und analysieren (vgl. ebenda, 
S. 67f). Vielmehr versehen Menschen Medieninhalte in einem aktiven Prozess und eingebettet in ihren sozio-kulturellen Kontext mit Bedeutung und eignen sich diese an. Dieser Ansatz betont die Handlungsfähigkeit von Menschen im Umgang mit Medien innerhalb ihres Alltags. Neben Folgehandlungen mit einem Medienprodukt, wie z.B. kognitive und emotionale Prozesse der Verarbeitung der rezipierten Inhalte oder die personale Kommunikation über Medieninhalte (vgl. Hepp, 2006, S. 245) meint Handlungsfähigkeit die sinnstiftende Herstellung von Bedeutung während des Mediennutzungsprozesses. Sowohl durch die Kommunikation über Medien in der Gruppe als auch über reflexive Prozesse beim Rezipieren und Handeln mit den Medien wird die Alltagswelt als Bezugsraum der Medienaneignung aktualisiert und verschiedene Lesarten der Medieninhalte werden ausgehandelt (vgl. Hepp, 2005, S. 72).

Auch im medienpädagogischen Diskurs wird Medienaneignung als Interaktion verstanden: erst im handelnden Umgang mit den Gegenständen seiner Umwelt eignen sich Subjekte diese an. Dabei handeln Subjekte sowohl stets eingebunden in ihren gesellschaftlichen Kontext und verändern diesen damit gleichzeitig (Theunert \& Schorb, 2004, S. 203). Aneignung meint dabei den "Prozess der Nutzung, Wahrnehmung, Bewertung und Verarbeitung von Medien aus der Sicht der Subjekte unter Einbezug ihrer - auch der medialen - Lebenskontexte" (ebenda, S. 210). So werden Medien aktiv in die sozialen Lebenskontexte einbezogen, indem vor dem Hintergrund der individuellen Biografie Angebote ausgewählt, Inhalten Aufmerksamkeit geschenkt oder diese vermieden werden sowie aktive Nutzungsmöglichkeiten genutzt werden (vgl. bspw. ebenda, S. 210).

Theunert \& Schorb (2010) beschreiben den Prozess der Aneignung vor dem Hintergrund der Mediatisierung als einen weitgehend informellen und selbstorganisierten Prozess, der vor allem auch in partizipativen und kollektiven (Online-) Kulturen stattfindet:

"Es sind nicht zuletzt die Heranwachsenden selbst, die ihre medialen Erlebnisse und Erfahrungen, ihre medienbezogenen Wissensbestände und Kompetenzen und ihre medialen Kommunikations- und Handlungspraktiken in die verschiedenen Lebensbereiche einbringen und sie zwischen ihren sozialen Bewegungsräumen hin und her tragen, sie in Auseinandersetzung und im Abgleich mit Gleichaltrigen und durch Beobachtung und Anregung von erwachsenen Bezugspersonen und Autoritäten ausbauen, variieren und verändern" (ebenda, S. 248).

Im Zuge der Mediatisierung verändern sich die reflexiven Aushandlungsprozesse. Im Web 2.0 kommt den aktiven Nutzungs-, Artikulations- und damit Partizipationsmöglichkeiten besondere Bedeutung zu. Beispielsweise in Online- 
Gemeinschaften, sozialen Netzwerken und anderen Gemeinschaftsformen (z.B. communities of practice) finden viele medialen - also verbale und audiovisuelle - Prozesse der Artikulation und Partizipation statt (Jörissen \& Marotzki, 2008, S. 203). Somit stellt das Internet für Jugendliche zunehmend einen kommunikativen Ort dar, "an dem Welt- und Selbstbezüge ausgeprägt und Wissensbestände modifiziert und erweitert werden" (Jörissen, 2007, S. 184). Die Hans-BredowStudie zum Heranwachsen Jugendlicher im Social Web (2009) stellt fest, dass sich ein reflektierter Umgang im Netz mit der Dauer und der Intensität der Nutzung zu entwickeln scheint (Schmidt, Paus-Hasebrink, \& Hasebrink, 2009, S. $10)$.

\subsection{Zweites Zwischenfazit: Medienaneignung bestimmt in hohem Maße den inter-generationalen Aushandlungs- prozess der Medienerziehung}

Der von Schleiermacher beschriebene dialektische Aushandlungsprozess zwischen den Generationen zu normativen Erziehungsfragen erfährt eine neue Qualität. Früher hieß es: was will die ältere Generation von der jüngeren und welchen Anforderungen will die jüngere Generation entsprechen? Heute tritt in der Medienerziehung das Problem auf, dass die ältere Generation zumeist gar nicht weiß, was sie wollen möchte, außer gegebenenfalls Veränderungen zu vermeiden. Dienste, die heute in hohem Maße die Mediennutzung dominieren, sind kaum zehn Jahre alt (Gründungsjahre von z.B. Google 1998, Wikipedia 2001, Facebook 2004, YouTube 2005, Twitter 2006). Von einem Wissensvorsprung der älteren Generation und insbesondere der bildungspolitischen Entscheidungsträger kann hier nicht die Rede sein. Während sich also kulturelle Handlungsmuster bedingt durch eine rasante Medieninnovation selbstorganisiert ausformen und somit zunehmend wichtige Rahmenbedingungen für die Entwicklung, Entfaltung und Sozialisation heranwachsender Generationen definieren, bedarf es eines inter-generationalen Diskurses um Normen und Ziele einer (notwendigen) Medienerziehung. Dieser Aushandlungsprozess ist unseres Erachtens ein Kernbereich der Medienbildung, die zwischen Erziehung und Aneignung vermitteln kann. 


\section{Medienbildung zwischen Medienkompetenz und Medienliteralität}

Die Anforderungen der aktiven Nutzung des Internets lassen sich nicht auf eine technische Bedienungskompetenz reduzieren bzw. stellen diese gar nicht in den Mittelpunkt einer entsprechenden Befähigung. Die partizipative Nutzung bedarf eher eines kreativen und kompetenten Umgangs mit netzbasierten multimodalen Medien, welcher von einigen Autoren insbesondere im anglo-amerikanischen Raum als New Media Literacy bezeichnet wird (vgl. Wolf, 2009). In der deutschsprachigen Diskussion taucht der Begriff Medien-Literalität kaum auf (Sacher, 2000, S. 99) und versteht sich als Erweiterung der Text-Literalität um weitere Medienformen.

\subsection{Medienliteralität und new media literacy}

Beispielhaft sollen im Folgenden drei Definitionsversuche vorgestellt werden, die das Spektrum der international zahlreichen Ansätze aufzeigen.

ICT Literacy: Für das vom US-amerikanischen Educational Testing Service (ETS) unter dem Namen iSkills vermarktete Assessment-Instrumentarium wird ICT literacy definiert als „.... the ability to use digital technology, communication tools, and/or networks appropriately to solve information problems in order to function in an information society. This includes the ability to use technology as a tool to research, organize, evaluate, and communicate information and the possession of a fundamental understanding of the ethical/legal issues surrounding the access and use of information." (ETS - Educational Testing Service, 2004, S. 11). Ähnliche Ansätze existieren in Europa in Form des Europäischen Computerführerscheins (ECDL Core, Advanced, ImageMaker und WebStarter), die noch mehr auf die technische Bedienfertigkeit ausgerichtet sind.

21st Century Literacy: Ein Beispiel für eine transdisziplinäre Orientierung an ästhetisch-gestalterischen, pädagogischen, medientheoretischen und kommunikationswissenschaftlichen Ansätzen wurde vom New Media Consortium definiert als „....the set of abilities and skills where aural, visual and digital literacy overlap. These include the ability to understand the power of images and sounds, to recognize and use that power, to manipulate and transform digital media, to distribute them pervasively, and to easily adapt them to new forms." (The New Media Consortium, 2005, S. 2). Ein an den Anforderungen einer veränderten Lebens- und Arbeitswelt orientiertes, deutlich weiter gefasstes Verständnis findet sich z.B. im Framework for 21st Century Learning der Partnership for 21st Century Skills (2007, S. 2), in welchem zwischen Information Literacy, Media 
Literacy sowie ICT Literacy differenziert wird. Diese bilden aber nur einen Aspekt neben Core Themes, Interdisciplinary Themes, Learning and Innovation Skills sowie Life and Carreer Skills.

New Media Literacy: Jenkins u. a. (2006, S. 3ff) postulieren eine sogenannte Kultur der Beteiligung ( prägt. Affiliations bezeichnen die Zugehörigkeit und das Aufbauen von onlinebasierten Netzwerken wie z.B. in Social Networking Diensten (z.B. studiVZ, Facebook, mySpace, Xing, LinkedIn) oder Onlinespielen bzw. Welten (z.B. World of Warcraft, Second Life). Expressions umfassen neue Formen des kreativen Ausdrucks, die insbesondere durch digitale Medien befördert werden, wie z.B. Sampling, Remixing, Skinning und Modding, Mashups, Fanzines und Fan Fiction. Collaborative Problem Solving spiegelt den Trend zum kollaborativen Arbeiten in vernetzten Medien wie z.B. Wikipedia wider. Circulations schließlich umfassen die aktive Beeinflussung von Informationsflüssen durch Blogging oder Podcasting, in denen Formen des Amateur Journalism die herkömmlichen Filtermechanismen der Massenmedien aushebeln.

Jenkins zweifelt jedoch an einem automatischen, beiläufigen Erwerb der dafür notwendigen Kompetenzen durch die von anderen Autoren als Net Generation bezeichnete Alterskohorte der mit dem Internet aufgewachsenen Kinder und Jugendlichen (zur empirischen Untermauerung dieser Kritik siehe Schulmeister, 2009) und fordert pädagogische und bildungspolitische Maßnahmen. Als zentrale Probleme bezeichnet Jenkins

(1) die ungleiche Beteiligung (Participation Gap), die durch heterogene Gelegenheiten, Erfahrungen und Kompetenzen entsteht;

(2) das Problem der mangelnden Transparenz (Transparency Problem) der Wirkungen der sich stetig ausdifferenzierenden Medienlandschaft auf die Wahrnehmung der Welt; sowie

(3) die ethische Herausforderung (Ethics Challenge) einer zunehmend fehlenden formalen beruflichen Ausbildung und Sozialisation zur Vorbereitung auf die zunehmend öffentlichen Rollen als Medienproduzenten und Mitglieder professioneller Gemeinschaften.

Auf Basis der Kultur der Partizipation und der sich daraus ergebenden Problemfelder fokussiert die Forschungsgruppe um Jenkins bei der Definition einer New Media Literacy - durchaus aufbauend auf traditionelle Basiskompetenzen wie Lesen, Schreiben und Rechnen - weniger auf technische Kompetenzen sondern eher auf kulturelle Kompetenzen und soziale Fertigkeiten, um innerhalb größerer Gemeinschaften mitwirken zu können und sich einzubringen (Jenkins u. a., 2006, S. 6). 
New Media Literacy umfasst in der Definition von Jenkins u. a. (2006, S. 55) folgende - als skills bezeichnete - Fähigkeiten und Fertigkeiten:

(1) Spielen (Play): das Experimentieren mit der eigenen Umwelt als eine Form des Problemlösens;

(2) Auftritt (Performance): alternative Identitäten zur Improvisation und Entdeckung nutzen;

(3) Simulation: realistische Modelle von dynamischen Prozessen interpretieren und konstruieren können;

(4) Aneignung (Appropriation): sinnvoll Ausschnitte aus Medien zu lösen (sampling) und neu kombinieren (remixing);

(5) Multitasking: bezeichnet die Fähigkeit, mehrere Tätigkeiten parallel oder abwechselnd in kurzen Zeitabschnitten durchzuführen. Dabei wird die Umgebung permanent überwacht, um sich nahezu automatisch auf hervorstechende Details zu fokussieren;

(6) Verteilte Kognition (Distributed Cognition): mittels kognitiven Werkzeugen die eigenen Verarbeitungskapazitäten verstärken;

(7) Kollektive Intelligenz (Collective Intelligence): Wissen mit Anderen teilen und Aufzeichnungen untereinander austauschen und vergleichen, um gemeinsame Probleme zu lösen, die für den Einzelnen zu schwer sind;

(8) Urteilsvermögen (Judgment): die Verlässlichkeit und Glaubwürdigkeit verschiedener Informationsquellen beurteilen;

(9) transmediale Orientierung (Transmedia Navigation): dem Verlauf von Geschichten und Information über Mediengrenzen folgen können;

(10) Vernetzung (Networking): Informationen suchen, zusammenzufassen und verbreiten;

(11) Verhandeln (Negotiation): zwischen verschiedenen Gemeinschaften wechseln und deren alternativen Normen und multiple Perspektiven wahrnehmen und respektieren.

Die von Jenkins genannten Fertigkeiten und Fähigkeiten werden nicht weiter in Oberbegriffe oder Bereiche zusammengefasst, sondern stehen nebeneinander. Sie beziehen sich auf neue und eher informelle und de-institutionalisierte Qualitäten der digitalen und vernetzten Medien: Interaktivität, soziale Vernetzung, Rekombinierbarkeit und Veränderbarkeit sowie Verlust von Autoritäten. 


\subsection{Zum Medienkompetenzbegriff}

Nach Durchsicht der verschiedenen Definitionsansätze der New Media Literacy drängen sich zwei kritische Fragen auf:

(1) Ist eine New Media Literacy nicht bereits im Kontext des Diskurses zur Medienkompetenz umfassend beschrieben worden oder bringt das Konzept neue Aspekte auf?

(2) Ist eine New Media Literacy nicht eine spezifische Form der Textliteralität und kann somit mit vorhandenen (Teil-)Konstrukten (z.B. Reading Literacy) beschrieben und verstanden werden?

Der Begriff der Literalität als eher bildungstheoretischer Begriff war zunächst dichotom geprägt (hat - hat nicht) und bezeichnete eine singuläre Fertigkeit, um in einer Gesellschaft zu funktionieren. Erst durch den Wechsel zu den New Literacy Studies wurden zusätzlich anthropologische und kulturübergreifende Perspektiven einbezogen und Literalität als ein Kontinuum verstanden (Linde, 2006, S. 96f). Bellmann (2007, S. 426) weist darauf hin, dass eine rein funktionalistische Sicht der Literalität (functioning in society) durch ein aktives und initiatives Verständnis von Literalität (participating in society) abgelöst worden ist. Eng verbunden mit dem Begriff der Literalität sind die Konzepte der Grundbildung und Alphabetisierung. Es geht bei der Literalität also vordringlich um ein Ermöglichen von Partizipation an Gesellschaft.

Aus der Sicht der Bildungsforschung findet dagegen eher ein aus der Expertiseforschung abgeleiteter Kompetenzbegriff Verwendung, der nach Weinert (2001) definiert ist als "die bei Individuen verfügbaren oder durch sie lernbaren kognitiven Fähigkeiten und Fertigkeiten, um bestimmte Probleme zu lösen, sowie die damit verbundenen motivationalen, volitionalen und sozialen Bereitschaften und Fähigkeiten, um die Problemlösungen in variablen Situationen erfolgreich und verantwortungsvoll nutzen zu können" (ebenda, S. 27f).

Vordringliches Ziel ist hier also die institutionalisierte Beschreibung bzw. Messung des individuellen Ausmaßes der Medienkompetenz, wobei kritisch anzumerken ist, dass gerade die nicht-kognitiven Aspekte der Definition nach Weinert bisher empirisch weitgehend unberücksichtigt geblieben sind.

Die Frage, warum im deutschsprachigen Diskurs zur Medienpädagogik das Konzept der Medienkompetenz mehr Resonanz gefunden hat als das der Medien-Literalität, ist wohl auf unterschiedliche Forschungstraditionen der jeweils beteiligten Wissenschaftsgemeinschaften zurückzuführen. Die Erwachsenenbildung beschäftigt sich in Deutschland zwar mit Literalität, dies allerdings zunächst ohne einen spezifischen Bezug zu den neuen Medien. Sie ist traditionell eher am 
Bildungsbegriff orientiert (Schlutz, 2001) und schließt erst allmählich und eher ambivalent an den Kompetenzdiskurs an (Rychen \& Salganik, 2003, S. 52f). Sozialwissenschaftlich orientierte Medienpädagogen wie die Bielefelder Forscher Baacke und Treumann dagegen haben das Thema bereits viel früher aus dem Blickwinkel der Kompetenzdebatte in den Diskurs eingeführt.

Ursprünglich sah Dieter Baacke die Medienkompetenz durch die Bausteine ,kommunikative Kompetenz' und ,Handlungskompetenz' zusammengesetzt. Der Ansatz der kommunikativen Kompetenz war inspiriert durch den Linguisten Noam Chomsky und meint die menschliche Fähigkeit sich unabhängig von äußeren Reizen grundsätzlich sprachlich kommunikativ mit der Umwelt auseinander setzen zu können. Mit Handlungskompetenz meinte Baacke „alle Formen der Weltbemächtigung und Weltveränderung [...], die zwar durch kommunikative Akte begleitet werden, aber über diese insofern hinausgehen, als hier Objekte, Gegenstände und Sachverhalte" tangiert werden (Baacke, 1999, S. 32). Des weiteren bestand Baacke darauf, dass Kompetenz kein statischer Besitz sei, den man erwerben könne. Im Begriff Kompetenz kommt vielmehr die anthropologische Haltung zum Ausdruck, dass Subjekte, indem sie sich äußern, artikulieren und handeln, als lebende Menschen mit ihren jeweiligen "Ichs in den unterschiedlichen Lebensäußerungen" anerkannt werden (ebenda).

Allerdings ist auch der Begriff Medienkompetenz nicht einheitlich definiert, wie Gapski (2001) zeigen konnte, der über einhundert verschiedene Definitionen analysiert hat. Ein zentrales und etabliertes Konzept zur Beschreibung von Medienkompetenz ist das Bielefelder Medienkompetenzmodell, welches sich an das Modell von Baacke (1996) anlehnt und verstanden wird als "individuelle Fähigkeit des Umgangs mit Medien, die eigenaktiv im Altersverlauf ausgebildet wird, gleichzeitig aber auch in Abhängigkeit zu sozialisatorischen Faktoren der Umwelt und zu spezifischen (pädagogischen) Förderungen und Forderungen steht" (Treumann u. a., 2007, S. 32). Nach Baacke gliedert sich Medienkompetenz in die vier Bereiche Medienkritik, Medienkunde, Mediennutzung und Mediengestaltung mit insgesamt neun Unterdifferenzierungen (ebenda, S. 33f):

Medienkritik: (a) Analyse bezeichnet das Erkennen und Erfassen von Zusammenhängen im Medienbereich; (b) Reflexion beschreibt die Fähigkeit, sich selbst und sein eigenes mediales Handeln zu analysieren; (c) Ethik umfasst die Fähigkeit, Werturteile über Medien und deren Inhalte unter Berücksichtigung der sozialen Konsequenzen von Medienentwicklung zu fällen.

Medienkunde: (a) Informative Medienkunde beinhaltet Kenntnisse über die Ausdifferenzierung der Medien; (b) Instrumentell-qualifikatorische Medienkunde beschreibt die technischen Fertigkeiten im Umgang mit Medien. 
Mediennutzung: (a) Rezeptiv-anwendende Nutzung umfasst Nutzungsgewohnheiten und Praktiken der Mediennutzung; (b) Interaktive Nutzung beschreibt den handelnden, aktiven Umgang bei der Mediennutzung.

Mediengestaltung: (a) Innovative Gestaltung bezieht sich auf Veränderungen oder Neuerungen im Medienbereich, also z.B. der Veränderung von Programmen; (b) Kreative Gestaltung als Form der ästhetischen Veränderung oder Gestaltungen wie z.B. die Gestaltung einer Website.

Während die so definierte Medienkompetenz sowohl den medienbezogenen Anteil der ICT Literacy als auch der 21st Century Literacy weitgehend abdeckt, geht die New Media Literacy nach Jenkins über diese hinaus. Der zentrale Unterschied ist dabei die Idee der Teilhabe an einer partizipativen (Online-)Kultur mittels digitaler Medien, die sich nur in Ansätzen (Interaktive Mediennutzung; Innovative und Kreative Mediengestaltung) und wenig ausdifferenziert im Bielefelder Medienkompetenzmodell wieder findet. Strukturell ergänzen sich die Ansätze. Eine tatsächliche Operationalisierung der von Jenkins vorgeschlagenen Kompetenzen fehlt jedoch und für empirische Untersuchungen wird diese erst noch zu entwickeln sein.

\subsection{New Media Literacy als moderne Form der Textliteralität und Sprachkompetenz}

Im Kontext der Diskussion zur Konzeptualisierung sprachlicher Kompetenzen werden diese in Lesefähigkeit, Schreibfähigkeit und Sprachbewusstheit bzw. Rezeption, Produktion und Bewusstheit ausdifferenziert (Jude \& Klieme, 2007). Im Bielefelder Medienkompetenzmodell finden sich dazu Parallelen in den Dimensionen Mediennutzung, Mediengestaltung und Medienkritik. Insgesamt ist die Sprachkompetenz deutlich funktional orientiert und wird zunehmend domänenspezifisch ausdifferenziert. Auch wenn Klieme u.a. in der Expertise zu Bildungsstandards versuchen, einen Bildungsbezug im Sinne einer lebenslangen Steigerung und Verfeinerung zu proklamieren (Klieme u. a., 2007, S. 65f), beschreibt das verwendete Kompetenzmodell klar eine Disposition von Personen, bestimmte Probleme bzw. Anforderungssituationen zu bewältigen, nicht aber einen lebenslangen Bildungsprozess zur Entwicklung einer selbständigen, kritischen und reflexiven Persönlichkeit. Insofern ist sowohl das Bielefelder Modell und insbesondere die New Media Literacy nach Jenkins deutlich bildungsorientierter zu verstehen, auch wenn sie dadurch sicherlich weniger leicht zu operationalisieren ist.

Innerhalb der PISA Studie wird - der Anlage der Studie als Large Scale Assessment geschuldet - lediglich der Teilbereich der reading literacy definiert als 
„....understanding, using and reflecting on written texts, in order to achieve one's goals, to develop one's knowledge and potential and to participate in society" (Programme for International Student Assessment, 2003, S. 108).

Von der generellen Idee her umfasst eine New Media Literacy die (schriftliche) Literalität, erweitert diese jedoch um drei konstitutive Elemente:

(a) Formen des technisch vermittelten, nicht-schriftlichen Ausdruckes wie z.B. Video, Podcasts aber auch Chat;

(b) Veränderungen der traditionellen Charakteristika schriftlicher Dokumente wie z.B. von überwiegend linearen Strukturen hin zur Nutzung von Vernetzungen (Hypermedia) oder von ausformulierter Schriftsprache hin zu Verkürzungen und zur Vermündlichung von Schriftsprache (Chat, Instant Messaging, Email);

(c) die Interaktion und Kommunikation innerhalb eines variabel-öffentlichen, nur eingeschränkt zu kontrollierenden sozialen Raumes.

Die Fähigkeit zum Schreiben und Lesen wird somit zu einem Prozess, der vom Individuum laufend in Bezug zur "Leserschaft" gebracht, passend zu den Regeln und Normen der jeweiligen Gemeinschaft gestaltet und die spezifischen, sich unterscheidenden Eigenschaften der jeweils verwendeten Medien nutzen muss. Dies geht über bisherige Konzepte der (Text-)Literalität hinaus. Die Frage allerdings, inwieweit Neue Medien sich z.B. auf den Schreibprozess auswirken und neue Formen der Sprachkompetenz als konstitutives Element einer eigenständigen New Media Literacy ausbilden, ist momentan empirisch noch nicht zu beantworten (Endres, 2004; MacArthur, 2006, S. 255ff).

Ebenso ist es Gegenstand eines aktuellen Diskurses, inwieweit das algorithmische Denken (vgl. bspw.: Hashagen, Persina, Schelhowe, \& Volkmann, 2008; Nake, 2001) und im Speziellen die Ko-Konstruktionstätigkeit mit digitalen Medien (z.B. Programmieren, Entwerfen intelligenter Textilien: http://dimeb.informatik.unibremen.de/eduwear) nicht nur analog zur numeracy eine eigene Ausprägung von Medienliteralität darstellt (Schelhowe u. a., 2009), sondern möglicherweise auch neue Handlungsräume (digitaler) Medienbildung darstellt (Zorn, 2010).

\subsection{Drittes Zwischenfazit: Das Beziehungsdreieck aus Medienbildung, Medienkompetenz und Medienliteralität} Der Medienkompetenzbegriff ist insbesondere wegen seiner mangelnden Anbindung an die bildungstheoretischen Begriffstradition umstritten. So argumentiert Kai-Uwe Hugger (2008, S. 96) mit Verweis auf Winfried Marotzki, dass Orientierungswissen und deren Ermöglichung die wesentliche Erweiterung von Medienbildung im Gegensatz zur Medienkompetenz sei. 


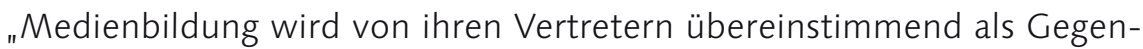
begriff zu Medienkompetenz dargestellt. Gefordert wird eine bildungstheoretische Grundlegung der Medienpädagogik. In nahezu allen Überlegungen wird ein latentes Unbehagen am Medienkompetenzbegriff festgestellt, weil dieser - wie die Kritiker meinen - vor allem der Vermittlung von instrumentellem Wissen bzw. Verfügungswissen entspreche, das also in erster Linie Qualifizierungsfunktion habe." (ebenda, S. 96f)
\end{abstract}

Hugger versteht deshalb Medienbildung und Medienkompetenz als zwei Seiten derselben Medaille (ebenda). Dies verbindet Medienbildung unseres Erachtens nach allerdings nur mit dem medienerzieherischen Tun. Wir würden deshalb aus dieser inhaltlichen Perspektive eher ein Dreieck zwischen Medienbildung, Medienkompetenz und zusätzlich Medienliteralität vorschlagen, welches nicht nur Orientierungs- und Verfügungswissen beinhaltet, sondern auch die Entfaltung von Partizipation.

\title{
5. Unsgestaltung als Kern der Medienbildung im Spannungs- feld des Lernens in formalen und informellen Kontexten
}

Die Etablierung von Online-Medien in weiten Teilen der Bildungslandschaft erweitert die Handlungsräume, stellt aber gleichzeitig auch neue Anforderungen an die Medienkompetenz der Lernenden. Es ist zu vermuten, dass der individuelle Erwerb einer Literalität neuer (Online-)Medien durch eine Affinität für Kommunikation, soziale Interaktion und kreative Gestaltung befördert wird. New Media Literacy stellt eine Erweiterung bekannter Basis- sowie Medienkompetenzen dar, insbesondere um Aspekte kultureller Kompetenzen und sozialer Fertigkeiten zur Partizipation in größeren (Online-)Gemeinschaften bzw. Kollektive. Eine verkürzte und oberflächliche Betrachtung im Sinne einer technischen Bedienungskompetenz verstellt den Blick auf die höchst anspruchsvolle Struktur einer partizipationsorientierten Literalität neuer Medien wie sie von der Forschungsgruppe um Jenkins exemplifiziert worden ist. Eine Annäherung der aufgezeigten Positionen deutet sich auch im Bericht der Expertenkommission des BMBF zur Medienbildung "Kompetenzen in einer digital geprägten Kultur" (Schelhowe u. a., 2009) an. Aus empirischer Sicht ist eine weitere Operationalisierung dieser Aspekte dringend notwendig, um deren Beitrag zum Bildungserfolg bzw. spezifische Bildungschancen und somit das Ausmaß möglicher Barrieren zur zukünftigen Bildungsteilnahme besser einschätzen zu können. 
Ein Großteil der tatsächlich stattfindenden Medienbildung, aktuell verstanden als reflexiver Selbstgestaltungsprozess, geschieht abseits der klassisch formalen und überwiegend institutionalisiert-pädagogisierten Kontexte. In unserer Analyse haben wir versucht, die immense Bedeutung informell-gemeinschaftlicher Prozesse der Medienbildung herauszuarbeiten, um diese in eine zentrale Position des kulturellen Medienhandelns zu rücken (siehe Abb. 1; Feld 4). Auch zeigt das Konzept der Medienliteralität in seiner Lesart nach Jenkins deutlich über bisherige Medienkompetenzdefinitionen hinausgehende Gestaltungsräume und Reflexionsanforderungen auf.

Stand der Fokus der traditionellen Medienpädagogik lange auf dem Individuum in seiner Auseinandersetzung entweder mit dem Massenmedium (z.B. wie nutze ich Fernsehen) oder in der individuellen Aufarbeitung der massenmedialen Elemente in einem weitgehend nicht-öffentlichen Umfeld (z.B. wie gestalte ich selbst eine Fernsehsendung, die aber keine Öffentlichkeit erreicht), so scheint es nötig, die Medienbildung um die Perspektive des reflexiv-relationalen Verhältnisses des Subjekts auch innerhalb von (Online-)Gemeinschaften bzw. Kollektiven (siehe Thomas \& Brown, 2011) zu erweitern.

Das Agieren in vernetzten (Online-)Medien stellt dabei den Begriff der Selbstgestaltung in Frage. Das Handeln im Social Web ist nämlich nur über die Relation zu Anderen zu verstehen. Der Kontext, die Bedeutung und der Sinn eines Beitrages im Netz wird erst über die sozial-inhaltliche Mitgestaltung anderer Benutzer/innen z.B. durch Trackbacks, Kommentare, Re-Tweets oder Like-Erwähnungen im Activity Stream von Profilen bestimmt. Ein Beitrag im Netz ist bezogen auf vorhergehende Beiträge und wird zum Bezugspunkt folgender Beiträge. Der einzelne Beitrag wird somit zu einem Teilbeitrag, der zusammen mit den anderen verknüpften Teilbeiträgen zum Metabeitrag emergiert. Durch die Bezugnahme z.B. in Form eines Kommentars verändert sich die Bedeutung des Beitragsgeflechtes. Die sowohl für die Medienerziehung als auch für die Medienaneignung bedeutsame individuelle Kreativität im Sinne von Medienproduktion wird durch die vernetzte Kommunikation zwangsläufig zu einer gemeinsamen Gestaltung.

Gleichzeitig ist die gemeinsame Bedeutungsfindung nicht unidirektional. Sie wirkt nicht nur in Richtung der Vergemeinschaftung, sondern auch zurück auf das einzelne Individuum. Folgerichtig müsste man nicht von einer Selbstgestaltung, sondern von einer medial vermittelten Unsgestaltung als Kern der Medienbildung sprechen.

Die Medienbildung hat dabei die zentrale Position, zwischen kulturellem Medienhandeln wie der Medienaneignung sowie der Entwicklung von Alltags- 
medienkompetenz und Medienliteralität einerseits und institutionell-unterstützenden Aneignungsformen wie Medienerziehung oder schulischer Medienkompetenzförderung andererseits zu vermitteln.

\section{Literatur}

Baacke, D. (1996). Medienkompetenz - Begrifflichkeit und sozialer Wandel. In A. V. Rein (Hrsg.), Medienkompetenz als Schlüsselbegriff (S. 112-124). Bad Heilbrunn: Klinkhardt.

Baacke, D. (1999). Medienkompetenz als zentrales Operationsfeld von Projekten. In D. Baacke, S. Kornblum, J. Lauffer, L. Mikos, \& G. A. Thiele (Hrsg.), Handbuch Medien: Medienkompetenz. Modelle und Projekte (S. 31-35). Bonn: Bundeszentrale für Politische Bildung.

Bachmair, B. (2009). Medienwissen für Pädagogen: Medienbildung in riskanten Erlebniswelten. Wiesbaden: VS Verlag für Sozialwissenschaften.

Bachmair, B., \& Burn, A. (2009). David Buckingham: Kindheit, Handlungsfähigkeit und Literalität. In A. Hepp, F. Krotz, \& T. Thomas (Hrsg.), Schlüsselwerke der Cultural Studies (S. 120-137). Wiesbaden: VS Verlag für Sozialwissenschaften.

Bellmann, J. (2007). Der Pragmatismus als Philosophie von PISA? Anmerkungen zur Plausibilität eines Deutungsmusters. Zeitschrift für Erziehungswissenschaft, 10(3), 421438. doi:10.1007/s11618-007-0044-5

Böhm, W. (1995). Theorie und Praxis: Eine Einführung in das pädagogische Grundproblem. Würzburg: Königshausen \& Neumann.

Endres, B. O. (2004). Ist Hypertext Text? In U. Kleinberger, F. Wagner, \& G. Kleinberger (Hrsg.), Neue Medien - Neue Kompetenzen? Texte produzieren und rezipieren im Zeitalter digitaler Medien (S. 33-48). Frankfurt: Peter Lang.

ETS - Educational Testing Service. (2004). Succeeding in the 21st Century. What Higher Education Must Do to Address the Gap in Information and Communication Technology Proficiencies. (National Higher Education ICT Initiative, Hrsg.). Abgerufen von http:/ /www.ets.org/Media/Tests/Information_and_Communication_Technology_Literacy/ ICTwhitepaperfinal.pdf

Feierabend, S., \& Rathgeb, T. (2009). JIM-Studie 2009. Jugend, Information, (Multi-) Media. Basisuntersuchung zum Medienumgang 12-bis 19-Jähriger. (Medienpädagogischer Forschungsverbund Südwest, Hrsg.). Stuttgart: Medienpädagogischer Forschungsverbund Südwest. Abgerufen von http://mpfs.de/fileadmin/JIM-pdf09/JIMStudie2009.pdf

Gapski, H. (2001). Medienkompetenz. Eine Bestandsaufnahme und Vorüberlegungen zu einem systhemtheoretischen Rahmenkonzept. Wiesbaden: Westdeutscher Verlag.

Hashagen, A., Persina, R., Schelhowe, H., \& Volkmann, G. (2008). "Der Schwarm" - Körpererfahrung und Algorithmik. In M. Herczeg \& M. C. Kindsmüller (Hrsg.), Mensch und Computer 2008: Viel Mehr Interaktion (S. 227-236). München: Oldenbourg. Abgerufen von http://mc.informatik.uni-hamburg.de/konferenzbaende/mc2008/konferenzband/mc2008_24_hashagen.pdf

Hepp, A. (2005). Kommunikative Aneignung. In L. Mikos \& C. Wegener (Hrsg.), Qualitative Medienforschung. Ein Handbuch (S. 67-79). Konstanz: UVK Verlagsgesellschaft. 
Hepp, A. (2006). Transkulturelle Kommunikation. Stuttgart: UTB.

Hirschauer, S. (2004). Praktiken und ihre Körper. Über materielle Partizipanden des Tuns. In K. H. Hörning \& J. Reuter (Hrsg.), Doing Culture: Neue Positionen zum Verhältnis von Kultur und sozialer Praxis (1. Aufl., S. 73-91). Bielefeld: Transcript.

Hugger, K. (2008). Medienkompetenz. In U. Sander, F. V. Gross, \& K. Hugger (Hrsg.), Handbuch Medienpädagogik (S. 93-99). Wiesbaden: VS Verlag für Sozialwissenschaften.

Jenkins, H., Purushotma, R., Clinton, K., Weigel, M., \& Robison, A. J. (2006). Confronting the Challenges of Participatory Culture: Media Education for the 21st Century. MacArthur. Abgerufen von http://www.newmedialiteracies.org/files/working/ NMLWhitePaper.pdf

Johnson, R. (1999). Was sind eigentlich Cultural Studies? In R. Bromley, U. Goettlich, C. Winter, G. Kreuzner, B. Suppelt, \& M. Haupt (Hrsg.), Cultural Studies: Grundlagentexte zur Einführung (S. 139-188). Lüneburg: Zu Klampen.

Jörissen, B. (2007). Informelle Lernkulturen in Online-Communities. Mediale Rahmungen und rituelle Gestaltungsweisen. In C. Wulf, B. Althans, G. Blaschke, N. Ferrin, M. Göhlich, B. Jörissen, R. Mattig, u. a. (Hrsg.), Lernkulturen im Umbruch. Rituelle Praktiken in Schule, Medien, Familie und Jugend (S. 184-219). Wiesbaden: VS Verlag für Sozialwissenschaften.

Jörissen, B., \& Marotzki, W. (2008). Neue Bildungskulturen im »Web 2.0«: Artikulation, Partizipation, Syndikation. In F. V. Gross, W. Marotzki, \& U. Sander (Hrsg.), Internet Bildung - Gemeinschaft (S. 203-225). Wiesbaden: VS Verlag für Sozialwissenschaften.

Jörissen, B., \& Marotzki, W. (2009). Medienbildung - Eine Einführung: Theorie - Methoden - Analysen. UTB (1. Aufl.). Stuttgart: Klinkhardt.

Jude, N., \& Klieme, E. (2007). Sprachliche Kompetenz aus Sicht der pädagogisch-psychologischen Diagnostik. In B. Beck \& E. Klieme (Hrsg.), Sprachliche Kompetenzen: Konzepte und Messung (S. 9-22). Weinheim: Beltz.

Klieme, E., Avenarius, H., Blum, W., Döbrich, P., Gruber, H., Prenzel, M., Reiss, K., u. a. (2007). Zur Entwicklung nationaler Bildungsstandards. Expertise im Auftrag des Bundesministeriums für Bildung und Forschung. Berlin: Bundesministerium für Bildung und Forschung (BMBF), Referat Öffentlichkeitsarbeit.

Krotz, F. (2007). Mediatisierung. Fallstudien zum Wandel von Kommunikation. Wiesbaden: VS Verlag für Sozialwissenschaften.

Linde, A. (2006). Alphabetisierung, Grundbildung oder Literalität? In A. Grotlüschen \& A. Linde (Hrsg.), Literalität, Grundbildung oder Lesekompetenz? Beiträge zu einer Theorie-Praxis-Diskussion (S. 90-99). Münster: Waxmann.

MacArthur, C. A. (2006). The Effects of New Technologies on Writing and Writing Processses. In C. A. MacArthur, J. Fitzgerald, \& S. Graham (Hrsg.), Handbook of Writing Research (S. 248-262). New York: Guilford Pubn.

Moser, H. (2010). Einführung in die Medienpädagogik. Aufwachsen im Medienzeitalter (5. Aufl.). Wiesbaden: VS Verlag für Sozialwissenschaften.

Nake, F. (2001). Das algorithmische Zeichen. In W. Bauknecht, W. Brauer, \& T. Mück (Hrsg.), Informatik 2001. Tagungsband der GI/OCG Jahrestagung 2001 (Bd. 2, S. $736-$ 742). Abgerufen von http://www.agis.informatik.uni-bremen.de/ARCHIV/Publikationen/Algor.ZeichenWienText.pdf

Partnership for 21st Century Skills. (2007). Framework for 21st Century Learning. Abgerufen von http://www.p21.org/documents/P21_Framework.pdf 
Programme for International Student Assessment. (2003). The PISA 2003 Assessment Framework - Mathematics, Reading, Science and Problem Solving Knowledge and Skills. OECD Publishing. Abgerufen von http://www.oecd.org/dataoecd/46/14/33694881.pdf Rychen, D. S., \& Salganik, L. H. (2003). A holistic model of competence. In D. S. Rychen \& L. H. Salganik (Hrsg.), Key competencies for a successful life and a well-functioning society (S. 41-63). Göttingen: Hogrefe \& Huber.

Sacher, W. (2000). Schule und Internet: Informations- und Wissensmanagement als zeitgemäße Bildungsaufgabe. In W. Marotzki, D. M. Meister, \& U. Sander (Hrsg.), Zum Bildungswert des Internet, Bildungsräume digitaler Welten (S. 97-113). Opladen: Leske + Budrich

Schelhowe, H., Grafe, S., Herzig, B., Koubek, J., Niesyto, H., Berg, A. V., Coy, W., u. a. (2009, März 12). Kompetenzen in einer digital geprägten Kultur. Medienbildung für die Persönlichkeitsentwicklung, für die Gesellschaftliche Teilhabe und für die Entwicklung von Ausbildungs- und Erwerbsfähigkeit. Bericht der Expertenkommission des BMBF zur Medienbildung. Bundesministerium für Bildung und Forschung. Abgerufen von http://www.bmbf.de/pub/kompetenzen_in_digital_kultur.pdf

Schlutz, E. (2001). Bildung. In R. Arnold, S. Nolda, \& E. Nuissl (Hrsg.), Wörterbuch Erwachsenenbildung (S. 48-51). Bad Heilbrunn: Klinkhardt.

Schmidt, J., Paus-Hasebrink, I., \& Hasebrink, U. (2009). Heranwachsen mit dem Social Web, Zur Rolle von Web 2.0 -Angeboten im Alltag von Jugendlichen und jungen Erwachsenen. Hans-Bredow-Institut fũr Medienforschung an der Universita t Hamburg Fachbereich Kommunikationswissenschaft der Universitãt Salzburg. Abgerufen von http://www.Ifm-nrw.de/fileadmin/Ifm-nrw/Aktuelle_Forschungsprojekte/ zusammenfassung_socialweb.pdf

Schmidt, J., Rohde, W., \& Hasebrink, U. (2009). Heranwachsen mit dem Social Web Tabellenband. Hans-Bredow-Institut für Medienforschung an der Universität Hamburg. Abgerufen von http://www.hans-bredow-institut.de/webfm_send/454

Schulmeister, R. (2009, Dezember). Gibt es eine Net Generation? Abgerufen von http:// www.zhw.uni-hamburg.de/uploads/schulmeister_net-generation_v3.pdf

Seipold, J., Rummler, K., \& Rasche, J. (2010). Medienbildung im Spannungsfeld alltäglicher Handlungsmuster und Unterrichtsstrukturen. In B. Bachmair (Hrsg.), Medienbildung in neuen Kulturräumen. Die deutschsprachige und britische Diskussion (S. 227241). Wiesbaden: VS Verlag für Sozialwissenschaften.

Spanhel, D. (2006). Handbuch Medienpädagogik 3: Erziehung- und Bildungsaufgaben in der Mediengesellschaft. Stuttgart: Klett-Cotta.

The New Media Consortium (Hrsg.). (2005). A Global Imperative - the report of the 21st century literacy summit. Abgerufen von http://www.adobe.com/education/pdf/ globalimperative.pdf

Theunert, H., \& Schorb, B. (2004). Sozialisation mit Medien: Interaktion von Gesellschaft - Medien - Subjekt. In D. Hoffmann \& H. Merkens (Hrsg.), Jugendsoziologische Sozialisationstheorie: Impulse furr die Jugendforschung, Jugendforschung (S. 203-235). Weinheim [u.a.]: Juventa-Verl.

Theunert, H., \& Schorb, B. (2010). Sozialisation, Medienaneignung und Medienkompetenz in der mediatisierten Gesellschaft. In M. Hartmann \& A. Hepp (Hrsg.), Die Mediatisierung der Alltagswelt, Medien - Kultur - Kommunikation (S. 243-254). Wiesbaden: VS Verlag für Sozialwissenschaften. 
Treumann, K. P., Meister, D. M., Sander, U., Burkatzki, E., Hagedorn, J., Kämmerer, M., Strotmann, M., u. a. (2007). Medienhandeln Jugendlicher. Mediennutzung und Medienkompetenz. Bielefelder Medienkompetenzmodell. Wiesbaden: VS Verlag für Sozialwissenschaften. Abgerufen von http://dx.doi.org/10.1007/978-3-531-90509-9

Tulodziecki, G. (2008). Medienerziehung. In U. Sander, F. V. Gross, \& K. Hugger (Hrsg.), Handbuch Medienpädagogik (S. 110-115). Wiesbaden: VS Verlag für Sozialwissenschaften.

Weinert, F. E. (2001). Leistungsmessung in Schulen - Eine umstrittene Selbstverständlichkeit. In F. E. Weinert (Hrsg.), Leistungsmessungen in Schulen (2. Aufl., S. 17-32). Weinheim und Basel: Beltz.

Wolf, K. D. (2009). Gender und New Media Literacy: Zugänge oder Barrieren beim ELearning? In M. K. W. Schweer (Hrsg.), Sex und Gender (S. 87-106). Frankfurt: Peter Lang.

Wolf, K. D. (2010). Metareflektion zu den Projekten Self und EduCamp. In H. Dürnberger, S. Hofhues, \& T. Sporer (Hrsg.), Offene Bildungsinitiativen. Münster: Waxmann.

Zorn, I. (2010). Konstruktionstätigkeit mit Digitalen Medien: eine qualitative Studie als Beitrag zur Medienbildung. Universität Bremen, Bremen. Abgerufen von http://nbnresolving.de/urn:nbn:de:gbv:46-diss000117767. 